\title{
Ein modulares Framework zur Modellierung, Konfiguration und Regelung von kooperativen Agrarprozessen
}

\author{
Frank Nordemann, M.Sc.; Franz Kraatz, M.Sc., MBA; \\ Prof. Dr. Heiko Tapken, Prof. Dr. Ralf Tönjes, \\ Hochschule Osnabrück, Fakultät Ingenieurwissenschaften und Informa- \\ tik, Albrechtstr. 30, 49076 Osnabrück, Deutschland
}

\begin{abstract}
Kurzfassung
Die Komplexität vieler Agrarprozesse nimmt aufgrund von technischem Fortschritt, steigenden rechtlichen Anforderungen und Nachweispflichten beständig zu. Prozessketten werden in Kooperation verschiedener Akteure (Landwirt, Lohnunternehmer, Dienstleister, digitaler Vermittler, Behörde) gemeinsam bearbeitet, dokumentiert und geprüft. Ein ökonomisch und ökologisch ressourceneffizientes Management der Prozessausführung stellt eine Herausforderung für alle Akteure dar. Dynamische Prozessveränderungen führen vielfach zu manuellen Eingriffen in die Prozessregelung, die kostenintensive Verzögerungen verursachen. Das Forschungsvorhaben OPeRAte entwirft und evaluiert neu gestaltete Konzepte und Mechanismen zur durchgehenden Organisation und Regelung kooperativer Agrarprozesse. Es werden konfigurierbare und wiederverwendbare Module identifiziert, die sich an Prozessparameter anpassen und in artverwandten Prozessen erneut verwenden lassen. Das OPeRAte-Framework ermöglicht die Zusammenführung aller beteiligten Akteure und Ressourcen (Maschinen, Sensoren, Aktoren, Endgeräte, Server, Daten, etc.) über offene Schnittstellen. Prozessinhaber sollen durch autonome Prozesskonfigurationen und -adaptionen entlastet und durch Visualisierungen zu effizienten Entscheidungen befähigt werden. Die Konzepte dieses Beitrags dienen als Diskussionsgrundlage zur Formulierung von flexiblen und erweiterbaren Lösungsstrategien für die Landtechnik.
\end{abstract}

\section{Motivation}

In der Landwirtschaft werden immer weniger Prozesse durch den eigentlichen Prozessinhaber, i.d.R. ein Landwirt, in Eigenarbeit durchgeführt. Die Komplexität von Prozessen (z.B. beim Precision Farming) und hohe Maschinenkosten haben zu kooperativen Arbeitsprozessen geführt, bei denen unterschiedliche Akteure Tätigkeiten übernehmen.

Bisher wird die Steuerung von Prozessen und der Austausch von Informationen vielfach manuell realisiert. Daten werden auf USB-Sticks oder per E-Mail zwischen Akteuren ausgetauscht, Steueranweisungen werden per Telefon durchgegeben. Zunehmend an Verbreitung gewinnen onlinebasierte Farmmanagementsysteme und Datendrehscheiben. Diese Systeme sind vielfach proprietär und erlauben keine bzw. nur eingeschränkte Kombinationen von Diensten verschiedener Anbieter. Weder eine autonome Orchestrierung (Zusammenstellung) und Konfiguration von Prozessen, noch eine dynamische Regelung zur Laufzeit ist möglich. In Zukunft werden die Anforderungen an kooperative Prozesse weiter steigen. Es müssen Datenschutz- und Datensicherheitsaspekte eingehalten werden. Auch Anforderungen an die Prozessdokumentation, z.B. bei der Flüssigmistdüngung, werden zunehmen. Optimierungen können nur unter kurzfristigem und akteurübergreifendem Austausch von Informationen realisiert werden. Beispielsweise werden in einem Precision-Farming-Prozess aktualisierte Ausbringkarten bei Wetteränderungen oder einem Wechsel des Düngers notwendig.

\section{Das OPeRAte-Forschungsprojekt}

Im dreijährigen Forschungsprojekt OPeRAte (Örchestrierung von Prozessketten für eine datengetriebene Ressourcenoptimierung in der Agrarwirtschaft und -technik) [1] kooperieren die Unternehmen 365FarmNet, ANEDO, FARMsystem und Kotte Landtechnik als Verbund 
mit der Hochschule Osnabrück. Die Förderung des Vorhabens erfolgt aus Mitteln des Bundesministeriums für Ernährung und Landwirtschaft (BMEL) aufgrund eines Beschlusses des deutschen Bundestages. Die Projektträgerschaft erfolgt über die Bundesanstalt für Landwirtschaft und Ernährung (BLE) im Rahmen des Programms zur Innovationsförderung. Seit dem Projektstart im Mai 2016 werden Verfahren für ein integriertes, ressourceneffizientes Farmmanagement, das anwendungsübergreifend die Erstellung und Optimierung von Prozessketten auf Geräte-, Daten- und Prozessebene unterstützt, erforscht.

Optimierungen von kooperativen Prozessen sollen akteur- und plattformübergreifend gestaltet werden. Die Entscheidungshoheit liegt weiterhin beim Prozessinhaber, der durch Funktionen zur Statusvisualisierung und Automation in Steuerungsentscheidungen unterstützt werden soll. Die Basis zur Realisierung der Vorhabensziele setzt das in der Entwicklung befindliche OPeRAte-Framework (siehe Abb. 1).

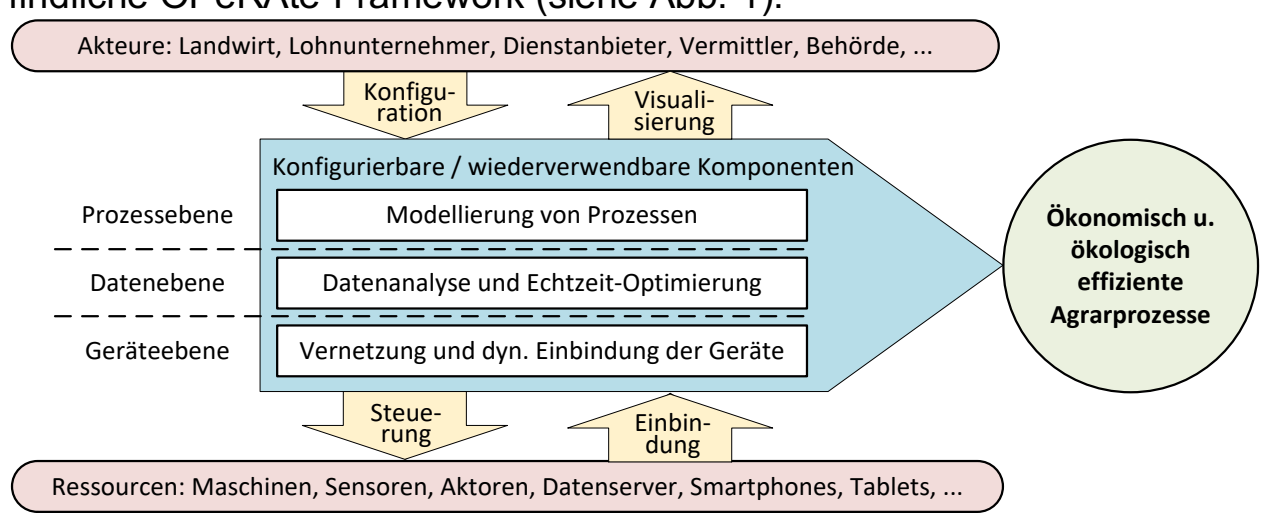

Abb. 1: OPeRAte-Framework zur Modellierung, Konfiguration und Regelung von kooperativen Agrarprozessen

Das OPeRAte-Framework verbindet modular-anpassbare und wiederverwendbare Komponenten der Ebenen Prozesse, Daten und Geräte miteinander (siehe Abb. 1). Über Schnittstellen zu Akteuren und Ressourcen lassen sich die Komponenten an den konkreten Anwendungsfall anpassen und zur Laufzeit ressourceneffizient optimieren.

\section{Modellierung von Prozessen in OPeRAte}

Die Beschreibung / Modellierung von Prozessen definiert Abläufe und Zusammenspiel von Prozessen und Prozessteilen. Sie soll mit möglichst geringem Aufwand erstellt, gleichzeitig aber auch flexibel auf spätere Veränderungen angepasst werden können. In der Landtechnik hat sich keine Sprache zur Modellierung von Prozessen etabliert. Der Austausch von Prozessen zwischen Systemen unterschiedlicher Anbieter ist nur schwer möglich. In diesem Kapitel wird das auf Flexibilität, Offenheit und Erweiterbarkeit ausgelegte OPeRAte-Konzept zur Prozessmodellierung vorgestellt.

\subsection{Flexibilität durch Prozessebenen}

Prozesse können sich in der Landtechnik mit der Bewirtschaftung von Feldern, der Viehzucht, der betrieblichen Maschinen- und Verbrauchsgüterplanung oder auch mit der rechtlich vorgeschriebenen Dokumentation von Arbeiten oder der Abrechnung von beauftragten Dienstleistungen befassen.

Flexibilität bei der Gestaltung von Prozessen wird in OPeRAte zunächst über die Aggregation von Subprozessen zu Prozessen realisiert. Ein Prozess kann aus beliebig vielen Subprozessen bestehen und gleichzeitig ein Subprozess eines übergeordneten Prozesses sein. Das nachträgliche Einfügen oder Austauschen von Prozessmodulen ist uneingeschränkt möglich. Zusätzlich tragen Prozessebenen zur Flexibilisierung bei. Ebenen erlauben eine bedarfsgerechte Sicht auf Prozesse und deren Zusammenspiel, bei der unnötige Detailinformationen abstrahiert werden. Vielfach ist eine Unterscheidung zwischen betrieblicher und aufgabenbezogener Steuerungsebene sinnvoll (siehe Abb. 2). Auf der betrieblichen Ebene 
werden landwirtschaftliche Prozesse wie Flüssigmistdüngung, Bodenbearbeitung und Aussaat gesteuert. Der Einsatz von Mitarbeitern, Maschinen und Verbrauchsgütern kann innerbetrieblich geplant werden. Konkrete Details, beispielsweise die einer Ernte, werden wiederum über die aufgabenbezogene Ebene in spezialisierten Subprozessen zusammengefasst. OPeRAte begrenzt weder die Anzahl möglicher Prozessebenen, noch werden spezielle Ebenen vorausgesetzt. Dadurch lassen sich weitere Ebenen, auch nachträglich, nach Bedarf hinzufügen. Abb. 2 visualisiert z.B. eine weitere, überbetriebliche Ebene, in der ein Prozess akteurübergreifend planen und steuern kann.

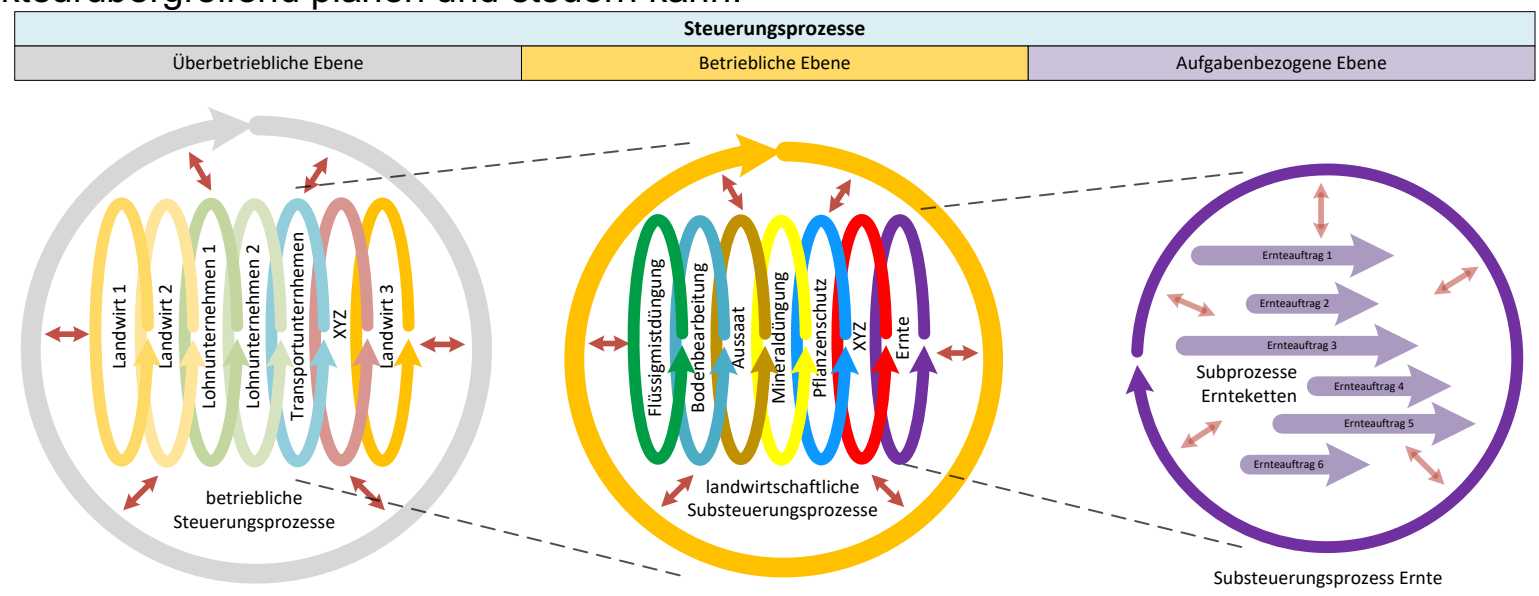

Abb. 2: Hierarchische Prozessebenen zur flexiblen und bedarfsgerechten Modellierung von Prozessen (hier: überbetrieblich, betrieblich, aufgabenbezogen)

\subsection{Vererbung und Erweiterbarkeit bei der Prozessmodellierung}

Prozesse weisen verschiedene Eigenschaften auf. Einige Eigenschaften teilen sie sich mit anderen Prozessen, andere unterscheiden sie wiederum von weiteren Prozessen. Typische Beispiele sind der Interaktionstyp (Einzelprozess / kooperativer Prozess) oder die Zeitkritik (zeitkritisch / nicht zeitkritisch). Die Prozessmodellierung soll größtmögliche Gestaltungsspielräume bei der Prozesserstellung erlauben und bereits definierte Eigenschaften bzw. Funktionen wiederverwenden können.

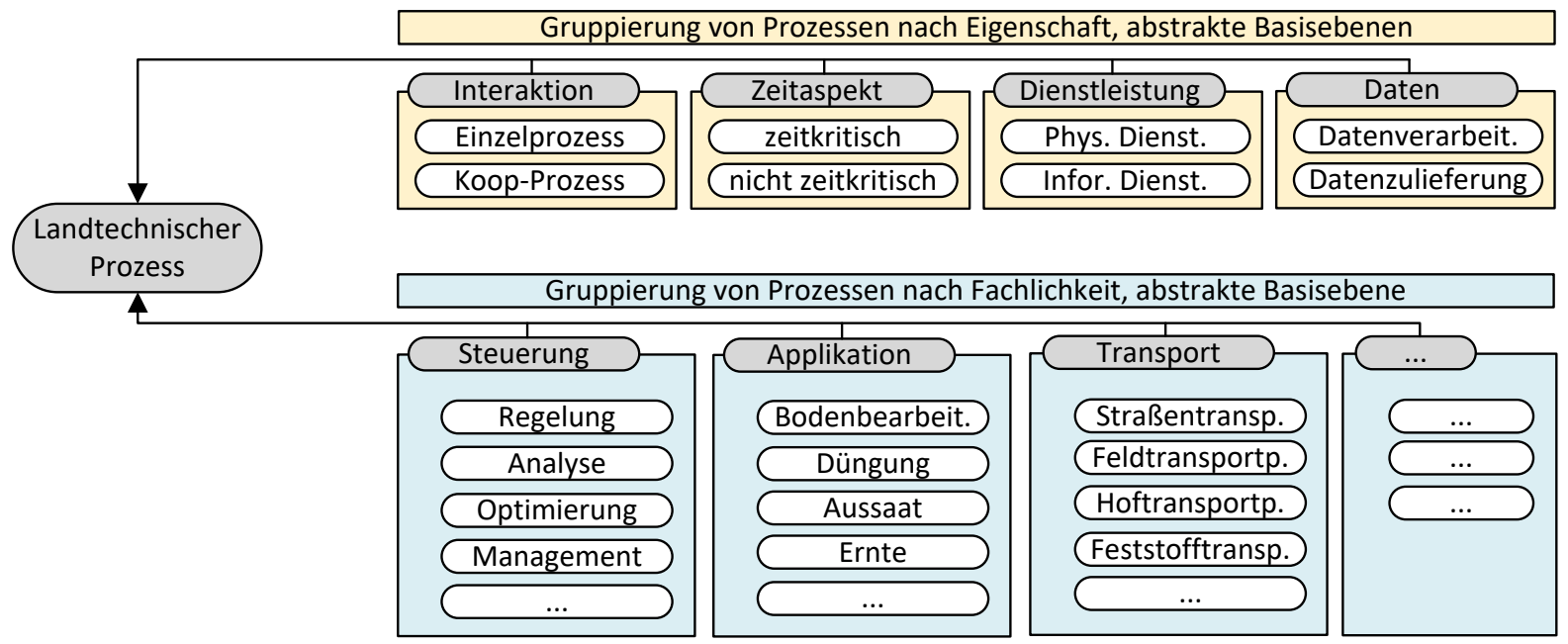

Abb. 3: OPeRAte-Vererbungskonzept zur Prozessmodellierung

Das Konzept des OPeRAte-Vorhabens spezialisiert Prozesse nach Fachlichkeit und Eigenschaft (siehe Abb. 3). Fachlichkeiten können Steuerungsprozesse, Applikationsprozesse oder Transportprozesse sein, d.h. Konkretisierungen eines allgemeinen landtechnischen Prozesses. Spezifische Ausprägungen dieser Prozessarten enthalten notwendige Erweite- 
rungen, teilen aber Funktionen (z.B. zeitliche Steuerungsmechanismen in Koop-Prozessen) der übergeordneten Prozessart. Die Gruppierung nach Eigenschaften verhindert Dopplungen bei der Prozessmodellierung. Dopplungen erhöhen den Wartungsaufwand und erschweren die maschinelle Lesbarkeit bzw. die Erstellung einer Wissensbasis, die für eine spätere autonome Prozessorchestrierung benötigt wird. Eigenschaften können bedarfsweise Prozessen zugewiesen werden. Ein Flüssigmisttransport kann z.B. als „zeitkritischer, kooperativer Prozess" gekennzeichnet werden und erhält dadurch entsprechende Funktionen zur Prozesssteuerung.

Das OPeRAte-Konzept zur Prozessmodellierung ist kein geschlossenes System. Prozesse und Prozessgruppierungen können nach Bedarf eingeführt und erweitert werden. Das Konzept erlaubt es, bestehende Beschreibungen von Prozessen und Objekten wieder zu verwenden. Ein landtechnischer Prozess kann als Konkretisierung eines allgemein beschriebenen und bereits definierten Prozesses gesehen werden (z.B. mittels der PSL [2]). Auch die AGROVOC-Ontologie [3] kann integriert werden. Hierüber kann z.B. Mineraldünger, der durch einen Managementprozess verwaltet werden soll, als bestehendes Objekt eingefügt werden (siehe Abb. 4). Auf ähnliche Weise können Beschreibungen des KTBL [4] oder von agroRDF [5] verwendet werden. Bei agroRDF liegen Informationen bereits als semantisches Modell in RDF-Form [6] vor, was Übertragung und Wiederverwendung vereinfacht.

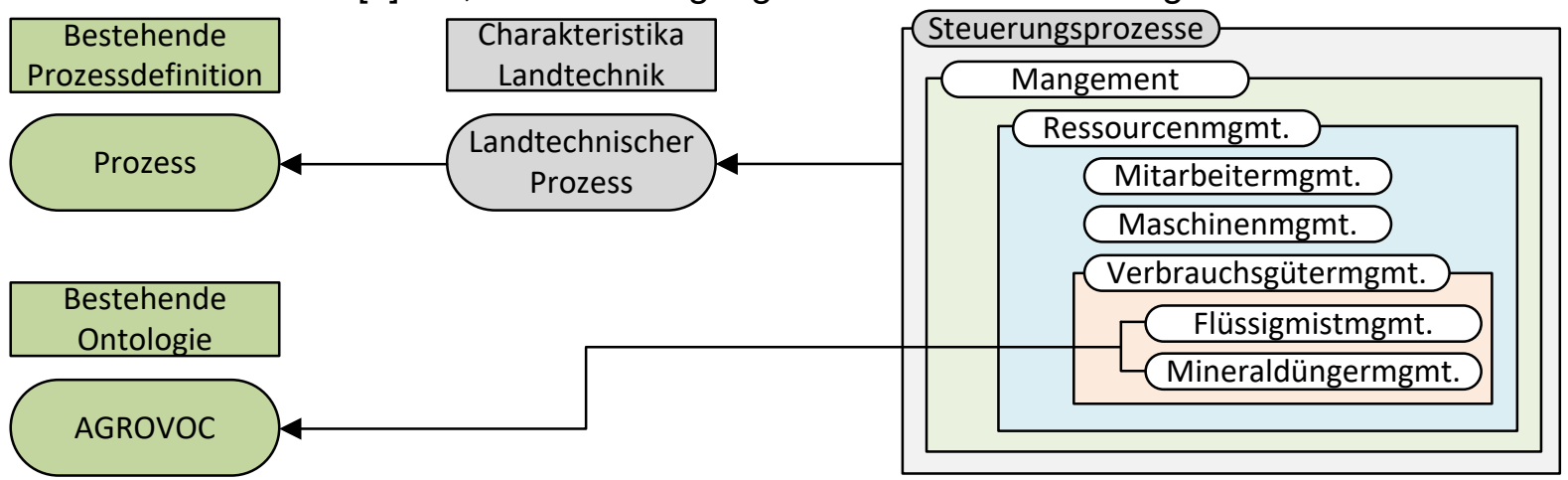

Abb. 4: OPeRAte-Prozessmodellierung zur Ableitung und Integration von bestehenden Konzepten, Beschreibungen und Regelwerken

\subsection{Semi-autonome Orchestrierung von Prozessen über Schnittstellen}

Mittels einer Gruppierung von Fachlichkeiten und Eigenschaften können Prozesse flexibel zusammengesetzt, gekapselt und um Funktionen erweitert werden. In Abb. 5 übernimmt der Prozess Flüssigmistausbringung Funktionen von den Prozessen Koop-Prozess, Optimierungsprozess und Steuerungsprozess. Weiterhin aggregiert der Prozess drei Subprozesse, die Ausbringung, Zubringung und Nährstoffanalyse implementieren. Orchestriert werden Prozesse über offene Schnittstellen, in denen Abhängigkeiten beschrieben werden (vgl. [7]). Schnittstellen bilden die Basis für eine semi-autonome Orchestrierung von Prozessen.

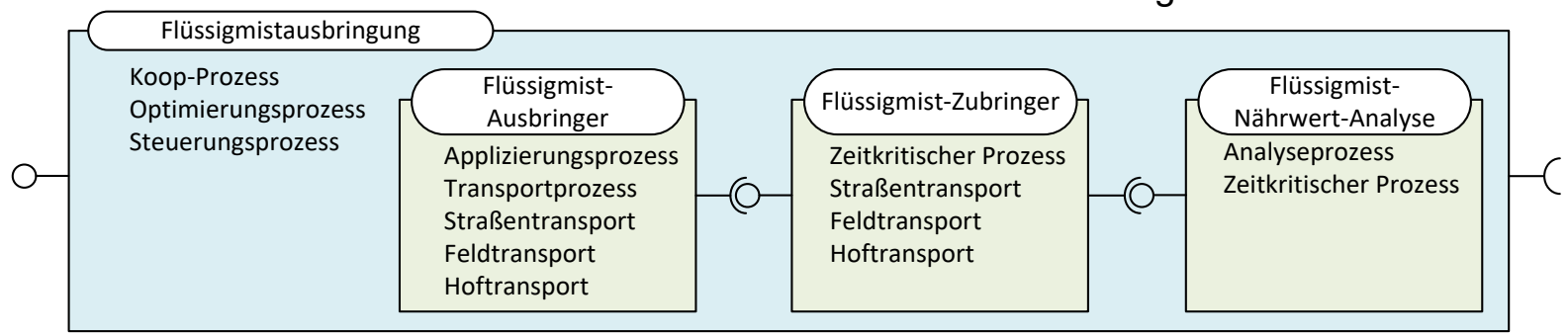

Abb. 5: Gestaltung eines Prozesses zur Flüssigmistausbringung durch Aggregation von Subprozessen, die auf der Ableitung von Fachlichkeiten und Eigenschaften basieren 


\section{Zusammenfassung und Ausblick}

Dieser Beitrag dient als Diskussionsgrundlage zur Lösung der derzeitigen und künftigen Herausforderungen bei der kooperativen Bearbeitung von landtechnischen Prozessen. Die OPeRAte-Konzepte erlauben eine flexible, bedarfsgerechte und auch nachträglich erweiterbare Modellierung und Orchestrierung von Prozessen. Strukturierende Prozessebenen sowie die Ableitung und Aggregation von Prozessen unterstützen die Erstellung und spätere Wiederverwendbarkeit von Prozessen und Funktionen.

Das OPeRAte-Forschungsvorhaben fokussiert neben der Prozessmodellierung weitere Aspekte kooperativer Agrarprozesse. Dazu gehören die Prozesskonfiguration zur Planungszeit sowie die dynamische Prozessregelung zur Laufzeit. Optimierungen werden durch den Einsatz von Big-Data-Algorithmen angestrebt, die auf anonymisierte Datenmengen unter Einhaltung von Datenschutz und Datensicherheit angewendet werden.

[1] OPeRAte-Forschungsprojekt, Hochschule Osnabrück et al. http://operate.edvsz.hs-osnabrueck.de (letzter Zugriff: 23.09.2016)

[2] Process Specification Language (PSL), National Institute of Standards and Technology. http://www.mel.nist.gov/psl (letzter Zugriff: 23.09.2016)

[3] AGROVOC Multilingual Agricultural Thesaurus, AIMS, FAO, 2016. http://aims.fao.org/vest-registry/vocabularies/agrovoc-multilingual-agricultural-thesaurus

[4] Kuratorium für Technik und Bauwesen in der Landwirtschaft e.V. (KTBL). http://www.ktbl.de (letzter Zugriff: 23.09.2016)

[5] Martini, D.; Schmitz, M.; Kunisch, M.: Datenintegration zwischen Standards in der Landwirtschaft auf Basis semantischer Technologien, Referate der 31. GILJahrestagung, Oppenheim, Februar 2011.

[6] Brickley, D.; Guha, R. V.: RDF Vocabulary Description Language 1.0: RDF Schema, W3C, 2004. http://www.w3.org/TR/rdf-schema (letzter Zugriff: 23.09.2016)

[7] Vogel, O. et al.: Software Architecture, Springer Verlag, Berlin-Heidelberg, 2011. 